\title{
Prevalência e fatores associados ao risco de quedas em idosos adscritos a uma Unidade Básica de Saúde do município de Natal, RN, Brasil
}

\author{
Prevalence and factors associated with the risk of falls \\ among the elderly registered in a primary healthcare unit \\ of the city of Natal in the state of Rio Grande do Norte, Brazil
}

Roberta Kelly Mendonça dos Santos ${ }^{1}$

Álvaro Campos Cavalcanti Maciel ${ }^{1}$

Heloísa Maria Jácome de Souza Britto ${ }^{1}$

Jackson Cláudio Costa Lima ${ }^{1}$

Túlio Oliveira de Souza ${ }^{1}$

${ }^{1}$ Departamento de Fisioterapia, Universidade Federal do Rio Grande do Norte. R. Joaquim Gregório s/n, Lagoa Nova. 59078900 Caicó RN Brasil. robertafisiojp@hotmail.com
Abstract The scope of the study was to analyze the prevalence and factors associated with falls among the elderly registered in a Primary Healthcare Unit (PHU) in Natal, State of Rio Grande do Norte. It involved a cross-sectional study conducted with 280 elderly individuals with data collected at the primary healthcare unit. The association between falls, recurrent falls and independent variables was assessed using bivariate analysis and Poisson regression with calculation of their respective prevalence ratios. It involved predominantly elderly females (68.2\%) with mean age of 71.6 years $( \pm 6.7)$, literate ( $54.6 \%)$, non-retired $(73.5 \%)$ and sedentary (87.1\%). $53.6 \%$ of the elderly recorded falls and $27.8 \%$ fell twice or more. The predictive model of falls included the female gender $(P R=1.81)$, osteoarticular diseases $(P R=$ $1.71)$ and balance impairment $(P R=0.88)$, while functional mobility $(P R=0.94)$, fear of falling $(P R=1.21)$ and balance deficit disorders $(P R=$ $0.80)$ constituted the definitive model of recurrent falls. A higher prevalence of single fall episodes was found and the associated factors included sociodemographic, health and physical performance variables. On the other hand, the occurrence of two or more falls were only associated with physical performance variables.

Key words Aging, Fall, Risk factors, Primary healthcare unit
Resumo $O$ objetivo deste artigo foi analisar a prevalência e os fatores associados a quedas em idosos adscritos a uma Unidade Básica de Saúde (UBS), no município de Natal/RN. Estudo transversal realizado com 280 idosos cujos dados foram coletados nas dependências da UBS. A associação entre os desfechos "queda" e "queda recorrente" $e$ as variáveis independentes foi verificada mediante análise bivariada e regressão de Poisson, com cálculo das respectivas razões de prevalências. Prevaleceram os idosos do gênero feminino (68,2\%), com média de idade de 71,6 anos ( $\pm 6,7)$, alfabetizados (54,6\%), não aposentados $(73,5 \%) e$ sedentários (87,1\%). Registraram queda 53,6\% dos idosos, porém $27,8 \%$ caíram duas ou mais vezes. O modelo preditivo de queda incluiu o gênero feminino $(R P=1,81)$, presença de doenças osteoarticulares $(R P=1,71)$ e comprometimento do equilíbrio $(R P=0,88)$, enquanto a mobilidade funcional $(R P=0,94)$, medo de cair $(R P=1,21)$ $e$ déficit de equilíbrio $(R P=0,80)$ compuseram o modelo final de quedas recorrentes. Encontrou-se maior prevalência de episódio único de queda e os fatores associados incluíram variáveis sociodemográficas, de saúde e performance física. Por outro lado, associaram-se a ocorrência de duas ou mais quedas apenas as variáveis de performance física. Palavras-chave Envelhecimento, Queda, Fatores de risco, Unidade Básica de Saúde 


\section{Introdução}

O envelhecimento populacional é um fenômeno mundial que configura-se como um dos eventos mais significativos da sociedade, adquirindo, ao longo dos anos, dimensões mais expressivas, particularmente nos países em desenvolvimento ${ }^{1}$. No Brasil, o segmento populacional representado pelos idosos é o que mais cresce. Projeções sinalizam que em 2025 o país ocupará o sexto lugar entre aqueles com maior número de idosos, quando aproximadamente $15 \%$ dos brasileiros terão idade igual ou superior a 60 anos, o que representa, em valores absolutos, 32 milhões de pessoas ${ }^{2}$.

Paralelamente às mudanças demográficas, ocorrem alterações nos padrões de morbimortalidade caracterizados pelo aumento de doenças crônico-degenerativas e de fatores que podem resultar em dependência e menor qualidade de vida, destacando-se a queda ${ }^{3}$. Definida como um deslocamento não intencional do corpo para nível inferior à posição inicial, a queda é causada por circunstâncias multifatoriais intrínsecas ou extrínsecas que comprometem a estabilidade ${ }^{4}$. Ela tem se revelado um problema de saúde pública pelas graves consequências que acarreta, sendo apontada como um marcador de perda da capacidade funcional, principal causa de hospitalização e mortes acidentais entre idosos 5 .

Além do caráter debilitante, a queda assume importância no cenário da saúde pública por apresentar alta prevalência, onerando o sistema de saúde na medida em que cresce a busca pela assistência médico-hospitalar para tratamento e reabilitação das lesões associadas ${ }^{6}$.

Estudos têm investigado as causas que levam o idoso a cair, porém, ainda há a necessidade de pesquisas no âmbito da atenção básica, tendo em vista o crescente número de idosos que se beneficiam da assistência oferecida pela Estratégia de Saúde da Família (ESF) e a necessidade de políticas preventivas que considerem as particularidades e as demandas específicas das sociedades ${ }^{7,8}$. Neste cenário, as equipes de saúde aparecem como aliadas ao cumprirem seu papel na identificação das situações de risco mais comuns às quais o idoso está exposto, bem como na elaboração de estratégias para o enfrentamento das mesmas $^{2,9}$.

O presente estudo teve por objetivo estimar a prevalência de quedas em idosos adscritos à uma Unidade Básica de Saúde (UBS) no município de Natal, e analisar os fatores associados.

\section{Metodologia}

O desenho desta pesquisa atendeu às recomendações do STROBE (Strengthening the Reporting of Observational Studies in Epidemiology) ${ }^{10}$ para estudos observacionais, sendo a investigação epidemiológica do tipo descritiva de corte transversal realizada com 280 usuários idosos cadastrados na UBS Felipe Camarão II, localizada no bairro Felipe Camarão, na cidade de Natal, RN. Trata-se de um bairro de classe econômica baixa, situado na zona oeste do município, compreendendo 663,40 ha de área territorial e uma população que abrange aproximadamente 50.997 habitantes, que representam $6,37 \%$ da população da cidade, tornando o bairro o terceiro mais populoso do município ${ }^{11}$.

Na UBS Felipe Camarão, estão inseridas quatro equipes ampliadas da ESF que prestam assistência a aproximadamente 4.000 famílias, abrangendo cerca de 15.000 moradores.

A população do estudo foi composta pela totalidade de idosos adscritos à UBS referida (n = 747), contudo, inicialmente, foi realizada uma consulta aos prontuários e à equipe de saúde para eleger os voluntários aptos a participar da pesquisa de acordo com os critérios de inclusão. Para serem incluídos, os voluntários deveriam ter 60 anos ou mais, não necessitar da ajuda de terceiros para a realização das atividades básicas de vida diária, tais como alimentar-se, banhar-se, vestirse e deambular, não utilizar dispositivos auxiliares para a marcha e não apresentar enfermidade, agravo à saúde e/ou deficiência visual, auditiva ou outra que implicasse em comprometimento em termos de comunicação ou execução dos testes na avaliação.

A coleta dos dados foi realizada nas dependências da UBS entre março e agosto de 2012, sempre pela mesma pesquisadora que realizou uma avaliação composta de questionário contendo informações sociodemográficas (idade, gênero, estado civil, prática de atividade física), de saúde por autorrelato, escalas e testes validados na versão brasileira para a avaliação da performance física e psicossocial em idosos, além do registro da ocorrência de queda e queda recorrente.

O histórico de quedas foi registrado por meio do questionamento sobre a ocorrência do agravo nos 12 meses que antecederam a pesquisa. Para tanto, foi realizado uma avaliação retroativa na qual o avaliado deveria lembra-se da quantidade de quedas sofridas no último ano: se nenhuma queda, uma queda, ou duas ou mais quedas. 

lisa o desempenho do idoso durante 14 tarefas representativas das atividades de vida diária, as quais incluem sentar, levantar, inclinar-se, entre outras. Cada item da escala contém 5 alternativas cujos escores variam de 0 a 4 pontos, sendo zero indicativo de incapacidade de realizar a tarefa e quatro indicativo da capacidade de executá-la com excelência. A pontuação total pode variar de 0 a 56 pontos, sendo que o maior valor representa o melhor desempenho no teste e, portanto, o melhor equilíbrio ${ }^{12}$.

O Timed Up and Go (TUG) foi utilizado para a avaliar a mobilidade funcional através da análise do equilíbrio sentado, transferências de sentado para de pé, estabilidade na deambulação e mudança do curso da marcha sem a utilização de estratégias compensatórias ${ }^{13}$.

O TUG quantifica, em segundos, a mobilidade através do tempo gasto para realizar a tarefa de levantar de uma cadeira padronizada com aproximadamente $46 \mathrm{~cm}$ de altura e apoio para os braços, percorrer 3 metros, retornar e sentar novamente ${ }^{14}$. Entre adultos saudáveis, o tempo médio para a realização da tarefa é de 10 segundos sendo que, conforme explicam ShumwayCook et al. ${ }^{13}$, idosos que executam o TUGT em tempo inferior a 20 segundos são considerados independentes nas AVD e apresentam velocidade de marcha suficiente para se locomover na comunidade. Aqueles que completam a tarefa em um tempo maior que 13,5 segundos apresentam maior risco de quedas.

O medo de cair foi avaliado por meio da Escala Internacional de Eficácia de Quedas Brasil (FES-I), a qual analisa, por meio de questionário, o quanto o indivíduo está preocupado com a possibilidade de cair se realizasse 16 atividades diárias distintas, cujos valores variam de 16 pontos para pessoas sem qualquer preocupação em cair a 64 pontos para aqueles com preocupação extrema ${ }^{15}$.

Por fim, o Mini Exame do Estado Mental (MEEM) foi utilizado para avaliar as funções cognitivas. O teste é composto por questões práticas agrupadas em sete categorias, cada uma delas planejada com o objetivo de avaliar funções cogniti-

vas específicas. $\mathrm{O}$ escore pode variar de 0 pontos, indicando maior grau de comprometimento cognitivo, até o máximo de 30 pontos, o qual corresponde a melhor capacidade cognitiva ${ }^{16}$.

A análise dos dados foi realizada por meio do software Statistical Package for the Social Sciences ${ }^{\circledR}$ (SPSS) versão 17.0, adotando-se o nível de significância de 5\% (p<0,05). A estatística descritiva foi realizada usando as medidas de distribuição (média, desvio padrão - DP, frequência absoluta e relativa), considerando as variáveis de interesse a fim de caracterizar a amostra.

$\mathrm{Na}$ abordagem analítica, inicialmente foi realizada a análise bivariada por meio do teste do Qui-quadrado de Pearson, para verificar a existência de associação entre variáveis categóricas; depois o teste $\mathrm{t}$ para amostras independentes, para comparação de médias entre grupos, considerando que todos os dados tiveram distribuição normal segundo o teste de Komolgorov-Sminorv (K-S).

Posteriormente, adotou-se o modelo de Regressão de Poisson para identificar as possíveis variáveis independentes associadas aos desfechos, com cálculo das respectivas razões de prevalência (RP). O desfecho utilizado foi a ocorrência de queda e de queda recorrente no ano anterior à pesquisa, enquanto que as variáveis testadas nos modelos foram: sexo, faixa etária, estado civil, escolaridade, prática de atividade física, ser ou não aposentado, o autorrelado da presença de patologias e comorbidade, além do uso de medicação contínua e de psicotrópicos.

Este estudo foi submetido à apreciação do Comitê de Ética e Pesquisa da Universidade Federal do Rio Grande do Norte, de acordo com as atribuições da resolução 196/96 do Conselho Nacional de Saúde, e foi aprovado. Todos os participantes receberam esclarecimentos e informações detalhadas acerca dos objetivos do estudo e dos procedimentos aos quais seriam submetidos e assinaram o Termo de Consentimento Livre e Esclarecido.

\section{Resultados}

Considerando os critérios de inclusão estabelecidos, 464 usuários idosos foram incluídos na pesquisa e distribuídos entre os Agentes Comunitários de Saúde (ACS) de suas respectivas áreas de abrangência para serem convocados para a avaliação. Destes, 12 mudaram de domicílio, 27 não foram encontrados em três visitas consecutivas realizadas em dias e horários diferentes, 24 
afirmaram não ser possível se ausentar do trabalho já que estavam ocupados nos dois expedientes, 12 haviam falecido, 66 não compareceram à avaliação, 29 se recusaram a participar do estudo e 14 não foram convidados pelos ACS. Todos estes idosos foram, portanto, excluídos do estudo de modo que 280 voluntários foram avaliados, compondo a amostra final.

Entre os 280 idosos estudados, $68,2 \%$ pertenciam ao gênero feminino e a maioria apresentava 70 anos ou mais (57,8\%), com média de idade de $71,6( \pm 6,7)$ anos. A prevalência de pelo menos um episódio de queda foi de $53,6 \%$, sendo que a maioria dos que caíram eram mulheres $(74,6 \%)$.

A descrição da amostra e a distribuição dos idosos que caíram ou não conforme características sociodemográficas e de saúde estão apresentadas na Tabela 1. Foi verificado que $141(50,3 \%)$ voluntários eram casados, $153(54,6 \%)$ alfabetizados, $206(73,6 \%)$ ainda trabalhavam e 244 $(87,1 \%)$ não praticavam nenhum tipo de atividade física. Quando questionados sobre o estado de saúde, $131(46,8 \%)$ responderam ter comorbidade, sendo o déficit visual a patologia de maior prevalência $(68,2 \%)$. A maioria dos idosos registrou o uso de medicação contínua (69\%).

As variáveis independentes de gênero, presença de comorbidade, doença osteoarticular e diabetes mellitus foram significativamente associadas ao desfecho queda no último ano.

A Tabela 2 mostra as características dos idosos com registro de queda recorrente. Dos voluntários avaliados, $27,8 \%$ apresentaram dois ou mais episódios de queda, sendo a maioria do gênero feminino $(80,7 \%)$. Estiveram associados a essa variável o gênero feminino $(\mathrm{p}<0,01)$ e o autorrelato de doenças osteoarticulares $(\mathrm{p}<0,01)$.

No que se refere às variáveis de performance física e psicossocial, a Tabela 3 indica que os idosos com registro de queda apresentaram menor desempenho nas atividades que envolvem o equilíbrio funcional avaliado pela EEB ( $\mathrm{p}<$ 0,01 ), além de baixa autoeficácia em evitar queda $(\mathrm{p}<0,01)$. Para quedas recorrentes, observa-se que houve diferença estatisticamente significativa nas médias obtidas em todas as variáveis de desempenho físico, de modo que os idosos que caíram duas ou mais vezes demonstraram menor desempenho nos escores obtidos na EEB ( $\mathrm{p}$ $<0,01)$, no TUG $(\mathrm{p}=0,03)$, FES - I $(\mathrm{p}<0,01)$ e $\operatorname{MEEM}(\mathrm{p}=0,03)$.

As variáveis selecionadas para a construção do modelo final de quedas e quedas recorrentes mediante análise de regressão logística múltipla estão descritas na Tabela 4. Após o ajustamento pelas variáveis independentes, permaneceram diretamente associados à ocorrência de quedas: $\mathrm{o}$ gênero feminino $\left(\mathrm{RP}_{\text {ajustada }}=1,81\right)$, o autorrelato de doenças osteoarticulares $\left(\mathrm{RP}_{\text {ajustada }}=1,71\right)$ e $\mathrm{o}$ baixo desempenho nas atividades avaliadas pela $\operatorname{EEB}\left(\mathrm{RP}_{\text {ajustada }}=0,88\right)$.

No que tange às quedas recorrentes, observou-se que os baixos escores na EEB e TUG constituíram-se fatores de risco para o agravo, já que os valores obtidos foram, respectivamente, BBS $\left(\mathrm{RP}_{\text {ajustada }}=0,08\right)$ e TUG $\left(\mathrm{RP}_{\text {ajustada }}=0,94\right)$. Com relação ao medo de cair, a análise mostra que a ausência de medo foi fator de proteção para a ocorrência do evento em questão $\left(R P_{\text {ajustada }}=1,21\right)$.

\section{Discussão}

O perfil sociodemográfico dos idosos estudados é semelhante ao encontrado em outras pesquisas brasileiras envolvendo idosos comunitários assistidos pelas $\mathrm{UBS}^{17,18}$, com amostra de indivíduos predominantemente do gênero feminino, alfabetizados, casados, sedentários, com acúmulo de diagnósticos autorreferidos e uso de medicação contínua.

A prevalência de quedas de $53,6 \%$ encontrada neste estudo foi maior que dados mostrados na literatura nacional ${ }^{18-20} \mathrm{e}$ internacional ${ }^{21-23}$. No entanto, este resultado pode ser comparado às pesquisas realizadas por Fabrício et al. ${ }^{24} \mathrm{e}$ Lopes et al. ${ }^{25}$, nas quais, respectivamente, $54 \%$ e $54,4 \%$ dos idosos brasileiros residentes em comunidade caíram.

Estudos populacionais realizados no Brasil têm registrado variações na prevalência de quedas $^{18,26}$, sendo estas mais acentuadas quando são recorrentes, principalmente na comparação entre países ocidentais e orientais, já que estes apresentam um percentual de quase o dobro dos primeiros $s^{6,27,28}$.

$\mathrm{Na}$ presente pesquisa, os dados seguem as alarmantes estatísticas dos países orientais e superam os achados na literatura nacional, uma vez que $27,8 \%$ afirmaram ter caído duas ou mais vezes, apontando a necessidade de estratégias e políticas preventivas locais ${ }^{22,29}$.

A ocorrência maior de quedas no sexo feminino corrobora outros estudos ${ }^{30,31}$, os quais as creditam ao fato das mulheres apresentarem maior fragilidade, maior prevalência de doenças crônicas e exposição às atividades domésticas que podem impor risco.

Diferente de pesquisas que evidenciaram associação entre idade e queda ${ }^{26,27}$, embora neste 
Tabela 1. Ocorrência de quedas e fatores associados segundo variáveis sociodemográficas e referentes à saúde dos idosos. Natal, RN, 2012.

\begin{tabular}{|c|c|c|c|}
\hline \multirow[t]{2}{*}{ Variáveis } & \multicolumn{2}{|c|}{ Queda no último ano } & \multirow[b]{2}{*}{ Valor $\mathrm{p}$} \\
\hline & Não & Sim & \\
\hline Idade $\mathrm{n}(\%)$ & & & 0,66 \\
\hline 60 a 69 anos & $53(40,8)$ & $65(44,0)$ & \\
\hline acima de 69 anos & $77(59,2)$ & $85(56,0)$ & \\
\hline Sexo n $(\%)$ & & & 0,01 \\
\hline Masculino & $51(39,2)$ & $38(25,3)$ & \\
\hline Feminino & $79(60,8)$ & $112(74,7)$ & \\
\hline Estado Civil n (\%) & & & 0,40 \\
\hline Solteiro/divorciado/viúvo & $68(52,3)$ & $71(47,3)$ & \\
\hline Casado/em união estável & $62(47,7)$ & $79(52,7)$ & \\
\hline Escolaridade n (\%) & & & 0,62 \\
\hline Não alfabetizado & $61(47,0)$ & $66(44,0)$ & \\
\hline Alfabetizado & $69(53,0)$ & $84(56,0)$ & \\
\hline Prática de Atividade Física n (\%) & & & 0,91 \\
\hline Não & $113(86,9)$ & $131(87,3)$ & \\
\hline $\operatorname{Sim}$ & $17(13,1)$ & $19(12,7)$ & \\
\hline Aposentado n (\%) & & & 0,36 \\
\hline Não & $99(76,1)$ & $107(71,3)$ & \\
\hline Sim & $31(23,8)$ & $43(28,7)$ & \\
\hline Comorbidades n (\%) & & & 0,02 \\
\hline Não & $70(53,8)$ & $61(40,7)$ & \\
\hline Sim & $60(46,1)$ & $89(59,3)$ & \\
\hline Déficit Visual n (\%) & & & 0,49 \\
\hline Não & $44(33,8)$ & $45(30,0)$ & \\
\hline Sim & $86(66,2)$ & $105(70,0)$ & \\
\hline Hipertensão Arterial n (\%) & & & 0,53 \\
\hline Não & $43(33,1)$ & $55(36,7)$ & \\
\hline Sim & $87(66,9)$ & $95(63,3)$ & \\
\hline Doença Osteoarticular n (\%) & & & 0,03 \\
\hline Não & $65(50,0)$ & $56(37,3)$ & \\
\hline Sim & $65(50,0)$ & $94(62,7)$ & \\
\hline Diabetes Mellitus n (\%) & & & 0,02 \\
\hline Não & $105(80,8)$ & $104(69,3)$ & \\
\hline Sim & $25(19,2)$ & $46(30,7)$ & \\
\hline Cardiopatias n (\%) & & & 0,32 \\
\hline Não & $113(86,9)$ & $124(82,7)$ & \\
\hline Sim & $17(13,1)$ & $26(17,3)$ & \\
\hline Uso de Medicação Contínua n (\%) & & & 0,69 \\
\hline Não & $42(32,3)$ & $45(30,0)$ & \\
\hline Sim & $88(67,7)$ & $105(70,0)$ & \\
\hline Uso de Medicação Psicotrópica n(\%) & & & 0,13 \\
\hline Não & $122(93,8)$ & $133(88,7)$ & \\
\hline Sim & $8(6,2)$ & $17(11,3)$ & \\
\hline
\end{tabular}

estudo o evento tenha sido maior entre os mais velhos, $56 \%$ naqueles com mais de 69 , a análise multivariada não mostrou associação entre tais variáveis. Os idosos de nossa pesquisa eram independentes, ativos na comunidade, e a maioria 
Tabela 2. Ocorrência de queda recorrente e fatores associados segundo variáveis sociodemográficas e referentes à saúde dos idosos. Natal, RN, 2012.

\begin{tabular}{|c|c|c|c|}
\hline \multirow[t]{2}{*}{ Variáveis } & \multicolumn{2}{|c|}{ Quedas Recorrentes } & \multirow[b]{2}{*}{ Valor $\mathrm{p}$} \\
\hline & Não & Sim & \\
\hline Idade n $(\%)$ & & & 0,56 \\
\hline 60 a 69 anos & $83(41,1)$ & $35(44,9)$ & \\
\hline acima de 69 anos & $119(58,9)$ & $43(55,1)$ & \\
\hline Sexo n $(\%)$ & & & $<0,01$ \\
\hline Masculino & $74(36,6)$ & $15(19,2)$ & \\
\hline Feminino & $128(63,3)$ & $63(80,8)$ & \\
\hline Estado Civil n (\%) & & & 0,94 \\
\hline Solteiro/divorciado/viúvo & $100(49,5)$ & $39(50,0)$ & \\
\hline Casado/em união estável & $102(50,5)$ & $39(50,0)$ & \\
\hline Escolaridade n (\%) & & & 0,33 \\
\hline Não alfabetizado & $88(43,6)$ & $39(50,0)$ & \\
\hline Alfabetizado & $114(56,4)$ & $39(50,0)$ & \\
\hline Prática de Atividade Física n (\%) & & & 0,99 \\
\hline Não & $175(94,6)$ & $69(72,6)$ & \\
\hline Sim & $10(5,4)$ & $26(27,3)$ & \\
\hline Aposentado n (\%) & & & 0,85 \\
\hline Não & $148(73,2)$ & $58(74,3)$ & \\
\hline $\operatorname{Sim}$ & $54(26,7)$ & $20(25,6)$ & \\
\hline Comorbidades n (\%) & & & 0,08 \\
\hline Não & $101(50,0)$ & $48(61,5)$ & \\
\hline Sim & $101(50,0)$ & $30(38,4)$ & \\
\hline Déficit Visual n (\%) & & & 0,17 \\
\hline Não & $133(65,8)$ & $58(74,3)$ & \\
\hline Sim & $69(34,1)$ & $20(25,6)$ & \\
\hline Hipertensão Arterial n (\%) & & & 0,63 \\
\hline Não & $133(65,8)$ & $49(62,8)$ & \\
\hline $\operatorname{Sim}$ & $69(34,1)$ & $29(37,1)$ & \\
\hline Doença Osteoarticular n (\%) & & & $<0,01$ \\
\hline Não & $105(51,9)$ & $51(65,4)$ & \\
\hline Sim & $97(48,0)$ & $27(34,6)$ & \\
\hline Diabetes Mellitus n (\%) & & & 0,11 \\
\hline Não & $46(22,8)$ & $25(32,0)$ & \\
\hline Sim & $156(77,2)$ & $53(68,0)$ & \\
\hline Cardiopatias n (\%) & & & 0,45 \\
\hline Não & $29(14,3)$ & $14(17,9)$ & \\
\hline Sim & $173(85,6)$ & $64(82,0)$ & \\
\hline Uso de Medicação Contínua n (\%) & & & 0,35 \\
\hline Não & $136(67,3)$ & $57(73,1)$ & \\
\hline Sim & $66(32,6)$ & $21(26,9)$ & \\
\hline Uso de Medicação Psicotrópica n(\%) & & & 0,15 \\
\hline Não & $15(7,4)$ & $10(12,8)$ & \\
\hline Sim & $187(92,6)$ & $68(87,2)$ & \\
\hline
\end{tabular}

significativa com um único evento queda após a análise ajustada, mostrando consonância com o que tem sido descrito na literatura ${ }^{21,32}$.

Bekibeli e Gureje $\mathrm{e}^{21}$, em estudo com 2.090 idosos, mostraram que a probabilidade de doenças osteoarticulares resultarem em queda é alta, sendo que a artrite reumatoide aumentou a chance do agravo quase duas vezes, o que pode ser explicado pelo comprometimento no tempo de reação da marcha, coordenação e equilíbrio decorrentes das dores articulares.

O equilíbrio funcional é amplamente avaliado pela EEB que foi considerada em alguns estu$\operatorname{dos}^{33,34} \mathrm{o}$ instrumento que melhor previu o risco de queda em idosos comunitários. Em nossa pesquisa, o menor desempenho conseguido na EEB 
Tabela 3. Análise das variáveis de performance física e psicossocial dos idosos com e sem registro de queda e queda recorrente no ano anterior à pesquisa. Natal, RN, 2012.

\begin{tabular}{|c|c|c|c|c|c|c|}
\hline \multirow{2}{*}{ Variáveis } & \multicolumn{3}{|c|}{ Queda no último ano } & \multicolumn{3}{|c|}{ Queda recorrente } \\
\hline & Sim & Não & Valor $\mathbf{p}$ & Sim & Não & Valor $\mathrm{p}$ \\
\hline $\mathrm{EEB}, \mu(\mathrm{DP})$ & $49,9( \pm 5,61)$ & $52,9( \pm 3,11)$ & $<0,01$ & $49,4( \pm 4,74)$ & $51,7( \pm 5,24)$ & $<0,01$ \\
\hline TUG, $\mu$ (DP) & $15,4( \pm 6,32)$ & $13,9( \pm 8,28)$ & 0,10 & $16,2( \pm 7,56)$ & $14,1( \pm 7,15)$ & 0,03 \\
\hline FES - I, $\mu(D P)$ & $32,5( \pm 10,67)$ & $26,8( \pm 9,67)$ & $<0,01$ & $34,5( \pm 10,02)$ & $28,3( \pm 10,44)$ & $<0,01$ \\
\hline MEEM, $\mu(\mathrm{DP})$ & $20,9( \pm 4,93)$ & $21,36( \pm 3,46)$ & 0,38 & $20,2( \pm 4,28)$ & $21,4( \pm 4,31)$ & 0,03 \\
\hline
\end{tabular}

$\mathrm{EEB}$ = Escala de Equilíbrio de Berg; TUG = Tumed Up and Go; FES - I = Escala Internacional de Eficácia de quedas; MEEM = Mini Exame do Estado Mental.

Tabela 4. Modelo final da associação entre as variáveis analisadas e a ocorrência de queda e queda recorrente. Natal, RN, 2013.

\begin{tabular}{|c|c|c|c|c|}
\hline \multirow[t]{2}{*}{ Variáveis } & \multicolumn{2}{|c|}{ Queda no último ano } & \multicolumn{2}{|c|}{ Queda recorrente } \\
\hline & RP ajustada (IC 95\%) & Valor $\mathbf{p}$ & RP ajustada (IC 95\%) & Valor $\mathrm{p}$ \\
\hline Sexo & & & -- & -- \\
\hline Masculino & 1 & & & \\
\hline Feminino & $1,81(1,21-2,67)$ & 0,03 & & \\
\hline DOA & & & -- & -- \\
\hline Não & 1 & & & \\
\hline Sim & $1,71(1,10-3,09)$ & 0,03 & & \\
\hline BBS & $0,88(0,77-0,96)$ & $<0,01$ & $0,80(0,75-0,85)$ & 0,03 \\
\hline FES - I & -- & -- & $1,21(1,12-1,31)$ & 0,02 \\
\hline TUG & -- & -- & $0,94(0,85-0,98)$ & 0,01 \\
\hline
\end{tabular}

EEB = Escala de Equilíbrio de Berg; TUG = Tumed Up and Go; FES - I = Escala Internacional de Eficácia de quedas; MEEM = Mini Exame do Estado Mental.

mostrou associação positiva para a ocorrência ( $\mathrm{p}$ $<0,01)$ e recorrência de queda $(\mathrm{p}=0,03)$ após análise multivariada.

Shumway-Cook et al. ${ }^{35}$ encontraram associação entre o agravo e o baixo escore na EEB. Estes autores sugerem que o teste é o melhor preditor isolado para a ocorrência de queda, havendo relação não linear entre a pontuação e o risco de cair. Cada ponto a menos aumenta o risco, sendo que, entre os escores 56 e 54, cada ponto a menos está associado ao aumento de 3\% a $4 \%$ na chance de queda. De 54 a 46, a alteração de um ponto aumenta o risco em $6 \%$ a $8 \%$; enquanto que abaixo de 36 pontos a chance de cair é de $100 \%$.

Outros estudos também encontraram risco de queda em idosos comunitários avaliados pela $\mathrm{EEB}^{36,37}$. Uma maneira de aumentar o equilíbrio funcional e diminuir o risco de queda é a prática de atividade física. Treinamentos físicos multi- modais que envolvam exercícios aeróbicos, força, potência, flexibilidade, coordenação e equilíbrio são efetivos para este fim ${ }^{7}$.

Embora a ocorrência de uma ou mais quedas tenha sido maior entre idosos sedentários $(87,3 \%$ e 72,6\% respectivamente), neste estudo não houve associação entre essas variáveis. Apesar disso, esse dado merece atenção, já que a inatividade predispõe à fragilidade, incapacidade e mortalidade, sendo considerada alvo de preocupação nas ações preventivas desenvolvidas no âmbito da atenção primária ${ }^{7,38}$.

Por ser um agravo de causa multifatorial, os riscos de queda e de queda recorrente aumentam linearmente com o número de fatores de risco. Tinetti e Williams ${ }^{39}$ mostraram que há uma probabilidade de $8 \%$ do idoso com nenhum risco cair e de $78 \%$ para aqueles que apresentam 4 fatores ou mais. Sai et al. ${ }^{40}$ também demonstraram 
associação entre o número de variáveis de risco e as quedas recorrentes, já que aqueles com uma única apresentaram menos fatores de risco do que os que caíram 2 ou mais vezes.

$\mathrm{Na}$ presente pesquisa, as medidas de desempenho físico dos idosos com quedas recorrentes foi aquém daqueles que caíram uma única vez, havendo diferença, estatisticamente significativa em todos os testes clínicos utilizados. Porém, além da $\mathrm{EEB}$, as variáveis que permaneceram no modelo final, estando associadas à recorrência de queda, foram o TUG e a FES-I.

O tempo de execução do TUG tem importância reconhecida na literatura, sendo que quando acima de 12 segundos, caracteriza o idoso como em alto risco ${ }^{41,42}$. A associação do TUG a uma única queda, como descrita em outros estudos ${ }^{43}$, não foi encontrada nesta pesquisa. Semelhante a este resultado, Sai et al. ${ }^{40}$ não mostraram diferença no teste de idosos que caíram ou não, mas verificaram que é preditor de duas ou mais quedas. Disso decorre a importância da marcha para identificar queda recorrente, sugerindo que os programas de prevenção envolvam atividades voltadas para o treino desta variável.

O medo de queda foi descrito por aqueles que sofreram tanto um quanto dois ou mais episódios, havendo associação desta variável somente com o evento queda recorrente.

Baixa autoconfiança em evitar queda é fator de risco independente para incapacidade e redução da mobilidade. Além disso, está relacionada com declínio da funcionalidade, aumento da fragilidade, depressão, ansiedade e perda do contato social, que repercutem no equilíbrio ${ }^{44}$. Para Morris et al. ${ }^{45}$, idosos que sofreram quedas recorrentes têm mais medo de cair do que aqueles que caíram uma única vez, o que pode explicar a associação encontrada no presente estudo.

A maioria dos idosos desenvolve o medo de cair novamente após sofrer queda com consequências graves, resultando em maior restrição das atividades e mobilidade, com consequente aumento cumulativo de prejuízos funcionais na predição de quedas recorrentes.

Os resultados desse estudo devem ser apreciados considerando algumas limitações, tais como a perda amostral que pode estar associada ao fato da coleta de dados ter sido desenvolvida nas dependências da UBS, o que pode ter desestimulado a participação daqueles que, por alguma razão, têm dificuldade de transporte, indisponibilidade ou pouca motivação em comparecer a eventos realizados na mesma. Esse fato também impossibilitou a avaliação dos riscos domiciliares e a abordagem do local da queda, importantes em estudos sobre o agravo.

É importante destacar a possibilidade de viés do respondente em relação à ocorrência de queda, já que os idosos podem ter omitido o evento por esquecimento, vergonha ou medo, sugerindo a relevância de estudos de seguimento abordando idosos no contexto das UBS.

Uma segunda questão diz respeito ao delineamento transversal, o qual impossibilita verificar uma associação de causalidade entre as variáveis. Além disso, a análise bivariada impede a consideração dos possíveis fatores de confusão que permeiam a determinação das quedas na amostra estudada. Desse modo, é importante destacar que este estudo pretendeu identificar o conjunto de variáveis que melhor contribui para identificar a ocorrência probabilística de queda e de queda recorrente, auxiliando a identificação de fatores de risco facilmente identificáveis pelos profissionais de saúde, o que permitirá uma abordagem rápida e efetiva que poderá diminuir sua ocorrência, beneficiando os idosos, seus cuidadores e o próprio Sistema de Saúde.

\section{Colaboradores}

RKM Santos trabalhou na concepção do estudo, definição do desenho da pesquisa, pesquisa bibliográfica, análise e interpretação dos dados, redação do artigo e revisão final para submissão. TO Souza trabalhou no levantamento dos dados, definição do desenho da pesquisa e concepção do estudo. HMJS Britto trabalhou na busca à literatura, levantamento e tabulação dos dados e concepção e revisão das tabelas. ACC Maciel trabalhou na análise e interpretação dos dados, concepção das tabelas, revisão crítica do artigo e revisão final para submissão. JCC Lima trabalhou no levantamento e tabulação dos dados e concepção do artigo. 


\section{Referências}

1. Cruz DT, Ribeiro LC, Vieira MT, Texeira MTB, Bastos RR, Leite ICB. Prevalência de quedas e fatores associados em idosos. Rev Saude Publica 2012; 46(1):138-146.

2. Veras SRP. Em busca de uma assistência adequada à saúde do idoso: revisão da literatura e aplicação de um instrumento de detecção precoce e de previsibilidade de agravos. Cade Saude Publica 2003; 19(3):705-715.

3. Matsumura BA, Ambrose AF. Balance in the elderly. Clin Geriatr Med 2006; 22(2):395-412.

4. Messias MG, Neves RF. A influência de fatores comportamentais e ambientais domésticos nas quedas em idosos. Rev Brase Geri e Gerontol 2009; 12(2):275-282.

5. Ribeiro AP, de Souza ER, Atie ES, de Souza AC, Schilitz AO. A influência das quedas na qualidade de vida de idosos. Cien Saude Colet 2008; 13(4):1265-1273.

6. Perracini MR, Ramos LR. Fatores associados a quedas em uma coorte de idosos residentes na comunidade. Rev Saude Publica 2002; 36(6):709-716.

7. Gillespie LD, Robestson MC, Gillespie WJ, et al. Interventions for preventing falls in older people living in the community. Cochrane Database of Syst Rev 2009; 4(6):1-193.

8. Gómes-Conesa A, Gama ZAS. Factores de riesgo de caídas em ancianos: revisión sistemática. Rev Saude Publica 2008; 42(5):946-956.

9. Brasil. Ministério da Saúde (MS). Secretaria de Atenção à Saúde. Política Nacional do Idoso. Brasília: MS; 1999.

10. Maltal M, Cardoso LO, Bastos FI, Magnanini MMF, da Silva CMFP. Iniciativa STROBE: subsídios para a comunicação de estudos observacionais. Rev Saude Publica 2010; 44(3):559-565.

11. Natal. Secretaria Especial do Meio Ambiente e Urbanismo (SEMURB). Departamento de Planejamento Urbanístico e Ambiental. Conheça melhor o seu bairro. Natal: SEMURB; 2009.

12. Miyamoto ST, Lombardi JI, Berg KO, et al. Brazilian version of the Berg balance scale. Braz J Med Biol Res 2004; 37(9):1411-1421.

13. Shumway-Cook A, Brauer S, Woollacott MH. Predicting the Probability for Falls in Community-Dwelling Older Adults Using The Timed Up \& Go Test. Phys Ther 2000; 80(9):896-903.

14. Podsiadlo D, Richardson S. The timed Up \& Go: A test of basic functional mobility for frail elderly persons. $J$ Am Geriatr Soc 1991; 39(2):142-148.

15. Camargos FOO, Dias RC, Dias JMD, Freire MTF. Adaptação transcultural e avaliação das propriedades psicométricas da Falls Efficacy Scale - Internacional em idosos brasileiros (FES - I-Brasil). Rev. Bras. Fisioter 2010; 14(3):237-243.

16. Bertollucci PHF, Brucki SMD, Campacci SR, et al. O mini-exame do estado mental em uma população geral: impacto da escolaridade. Arq Neurol 1994; 52(1):1-7.

17. Cruz DT, Ribeiro LC, Vieira MT, Texeira MTB, Bastos RR, Leite ICG. Prevalência de quedas e fatores associados. Rev Saude Publica 2012; 46(1):138-146.

18. Dantas EL, de Brito GEG, Lobato IAF. Prevalência de quedas em idosos adscrtios à Estratégia de Saúde da Família do município de João Pessoa, Paraíba. Rev APS 2012; 15(1):67-75.
19. Pereira GN, Morschi P, Lopes DGC, Trevisan MD, Ribeiro A, Navarro JHN, Bós DSG, Vianna MSS, Bós AJG. Fatores socioambientais associados à ocorrência de quedas em idosos. Cien Saude Colet 2013; 18(12):350735014.

20. Pinho TAM, Silva AO, Turas LFR, Moreira MASP, Gurgels SN, Smiths AAF, Bezerra VP. Avaliação do risco de quedas em idosos atendidos em Unidade Básica de Saúde. Rev Esc Enf USP 2012; 46(12):320-327.

21. Bekibele CO, Gureje O. Fall incidence in a population of elderly persons in Nigeria. Gerontology 2010; 56(3):278-283.

22. Milat AJ, Watson WL, Monger C, Barr M, Giffin M, Reid M. Prevalence, circumstances and consequences of falls among community-dwelling older people: results of the 2009 NSW Falls Prevention Baseline Survey. NSW Public Health Bulletin 2011; 22(3-4):60-68.

23. Halil M, Ulger Z, Cankurtaran M, Shorbagi A, Yavuz BB, Dede D, Ozkayar N, Ariogul S. Falls and the elderly: is there any difference in the developing world? A cross-sectional study from Turkey. Arch Gerontol Geriatr 2006; 43(3):351-359.

24. Fabricio SCC, Rodrugues RAP, da Costa Junior ML Causas e consequências de quedas de idosos atendidos em hospital público. Rev Saude Publica 2004; 38(1):9397.

25. Lopes KT, Costa DF, Santos LF, Castro DP, Bastone AC. Prevalência do medo de cair em uma população de idosos da comunidade e sua correlação com mobilidade, equilíbrio dinâmico, risco e histórico de quedas. Rev. Bras. Fisiot 2009; 13(3):223-229.

26. Siqueira FV, Facchini LA, Piccino RX, Tomasi E, Thumé E, Silveira DS, Vieira V, Hallal PC. Prevalência de quedas em idosos e fatores associados. Rev Saude $\mathrm{Pu}$ blica 2007; 41(5):749-756.

27. Chu LW, Chi I, Chiu AY. Incidence and predictors of falls in the chinese elderly. Ann Acad Med Singap 2005; 34(1):60-72.

28. Salvà A, Bolíbar I, Pera G, Arias C. Incidence and consequences of falls among elderly people living in the community. Med Clin 2004; 122(5):172-176.

29. Santer T, Bruggemann CFVP, Silva OM. Prevalência de quedas entre idosos frequentadores das unidades básicas de saúde do município de palmitos, Santa Catarina, e fatores associados. Rev Saude Publica 2012; 5(2):3243.

30. Fhon JRS, Wehbe SCCF, Vendruscolo TRP, Stackfleth $\mathrm{R}$, Marques S, Rodrigues RAP. Quedas em idosos e sua relação com a capacidade funcional. Rev. Latino-Am Enfermagem 2012; 20(5).

31. Pereira AA, Ceolim MF, Neri AL. Associação entre sintomas de insônia, cochilo diurno e quedas em idosos da comunidade. Cad Saude Publica 2013; 29(3):535-546.

32. Sachetti A, Vidmar MF, da Silveira MM, Schneider RH, Wibelinger LM. Risco de queda em idosos com osteoporose. Rev Bras Cienc Saude 2010: 8(24):23-25.

33. Shumway-Cook A, Woollacott MH. Controle Motor: teoria e aplicações práticas. 2a ed. São Paulo: Manole; 2003. 
34. Whitney SL, Poole JL, Cass SP. A review of balance instruments for older adults. Am J Occup Ther 1998; 52(8):666-671.

35. Shumway-Cook A, Balwin M, Polissar NL. Gruber W. Predicting the probability for falls in community-dwelling older adults. Phys Ther 1997; 77(8):812819.

36. Donat H, Özcan A. Comparison of the effectiveness of two programmes on older adults at risk of falling: unsupervised home exercise and supervised group ex-

37. ercise. Clinic Rehabil 2007; 21(3):273-283.

Alves NB, Scheicher ME. Equilíbrio postural e risco para queda em idosos da cidade de Garça, SP. Rev Bras

38. Geriatr Gerontol 2011; 14(4):763-768.

Cooper R, Kuh D, Cooper C, Gale CR, Lawlor DA, Mattheuws F, Hardy R. Falcon and Halcyon Study Teams. Objective measures of physical capability and subsequent health: a systematic review. Age Ageing 2011; 40(1):14:23

39. Tinetti ME, Williams CS. The effect of falls and falls injuries on functioning in community-dwelling older persons. J Gerontol 1998; 53(2):429-434.

40. Sai AJ, Gallagher JC, Smith LM, Logsdon S. Fall predictors in the community dwelling elderly:A cross sectional and prospective cohort study. J Musculoskelet Neuronal Interact 2010; 10(2):142-150.

41. Shumway-cook A, Brauer S, Woollacott MH. Predicting the Probability for Falls in Community-Dwelling Older Adults Using The Timed Up \& Go Test. Phys Ther 2000; 80(9):896-903.
42. Steffen TM, Hacker TA, Mollinger L. Age-and gender-related test performance in community-dwelling elderly people: Six-Minute Walk Test, Berg Balance Scale, Timed Up \& Go Test, and gait speeds. Phys Ther 2002; 82(2):128-137.

43. Thrane G, Joakimsen RM, Thornquist E. The association between timed up and go test and history of falls: The tromso study. BMC Geriatr 2007; 7(1):1-7

44. Brouwer BJ, Walker C, Rydahl SJ, Culham EG. Reducing fear of falling in seniors through education and activity programs: a randomized trial. J Am Geriatr Soc 2003; 51(6):829-834.

45. Morris M, Osborne D, Hill K, Kendig H, Lundgren-Lindquist B, Browning C, Reid J. Predisposing factors for occasional and multiple falls in older Australians who live at home. Aust J Physiother 2004; 50(3):153-159.

Artigo apresentado em 18/03/2015

Aprovado em 21/04/2015

Versão final apresentada em 23/04/2015 